\title{
C.S. Peirce
}

\section{Sa pensée pour}

une représentation

des connaissances et

> Modéliser les connaissances et le raisonnement médical peut être un projet épistémologique à I'heure où la médecine atteint un statut scientifique. Par ses travaux sur la sémiotique, le raisonnement abductif et le pragmatisme, C.S. Peirce (1839-1914) propose une série de solutions originales. Celles-ci peuvent rendre compte d'une grande partie de l'activité médicale dans divers domaines, comme l'évaluation des étudiants, les bases de connaissances et les systèmes experts, les langages de descriptions formelles (ontologies), la médecine fondée sur les preuves. Par ce texte, nous souhaitons présenter aux acteurs de la biomédecine cette pensée complexe mais éclairante sur une profession qui utilise désormais toutes les ressources les plus modernes de l'ingénierie des connaissances. <

Charles Sanders Peirce (1839-1914), philosophe logicien [1], est le fondateur de la sémiotique ${ }^{1}$, du raisonnement par abduction et du courant pragmatiste [2]. Plusieurs médecins [3-5] ont souligné l'originalité de sa pensée et l'adaptation qui peut en être faite en pratique médicale. Notre souhait ici est, au-delà de son apport pour la sémiologie médicale, de montrer comment l'ensemble de son système de pensée peut aider à modéliser la représentation des connaissances et le raisonnement médical.

Le système de pensée de Peirce couvre les aspects les plus fondamentaux de la médecine. II permet d'apporter un regard cohérent sur cette discipline. II permet également de dégager les éléments de connaissances qui lui sont propres, offrant le point de départ à sa modélisation. Celle-ci trouve son aboutissement dans le traitement informatique des données médicales, pour le raisonnement formel au travers des ontologies ${ }^{2}$, pour l'évaluation des connaissances au travers des tests de

${ }^{1}$ La sémiotique étudie le processus de signification, c'est-à-dire la production, la codification et la communication de signes.

${ }^{2}$ Ensemble structuré des termes et concepts représentant le sens d'un champ d'informations.

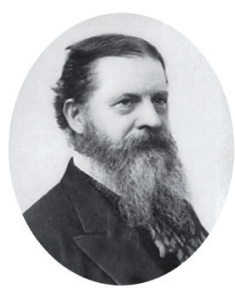

concordance des scripts ${ }^{3}$ [6], pour l'analyse de données apportées par le big data grâce aux logiciels abductifs et, enfin, pour l'evidence-based medicine qui veut que le raisonnement soit pragmatiquement vérifié au travers d'une action [7].

\section{Les catégories de Peirce : un rapport au monde différent}

Dans la lignée d'Aristote [8] et de Kant [9], Peirce a cherché à rendre compte de toute l'expérience humaine en se fondant sur le principe de catégories fondamentales. II n'en retiendra que trois qu'il nommera «priméité », «secondéité » et « tiercéité » ${ }^{4}$.

La priméité ne peut être définie par sa qualité intrinsèque puisqu'alors définie par rapport à son existence au sein de son environnement, elle sortira de cette catégorie. La définition de l'objet isolé dans le monde repose seulement sur son essence sans référence à quoi que ce soit d'autre. Cet objet n'a donc pas de poids puisque la gravité suppose la présence d'une planète (et donc d'un autre objet) ; il n'a pas de couleur puisque la lumière n'existe pas. Cet artéfact accepte le prédicat à une place «est un ... »: par exemple, «est un objet inanimé», «est un homme». Au sommet de la taxonomie ${ }^{5}$, se trouve la qualité générale et, au plus bas, les qualités non réductibles. Pour illustrer les relations existant entre objets qui peuvent être appliquées à la médecine, nous

\footnotetext{
${ }^{3}$ Les tests de concordance de script (TCS) sont des outils standardisés qui permettent d'évaluer le raisonnement clinique du professionnel qui le réalise et notamment sa capacité à prendre des décisions pertinentes dans des cas complexes, ou en situation d' « incertitude », en les confrontant aux avis d'un panel d'experts.

${ }^{4}$ Pour un médicament : la priméité est sa formule chimique, la secondéité est un effet souhaité ou non chez un patient, et la tiercéité, les observations des effets des médicaments (éventuellement réunies dans la notice).

${ }^{5}$ On parle ici de classification.
}

${ }^{1} \mathrm{CHU}$ de Martinique, pôle
imagerie medicale, 97261 Fort de France, France. campus Villejean, 2, avenue du professeur Léon Bernard, regis.duvauferrier@ chu-martinique.fr 


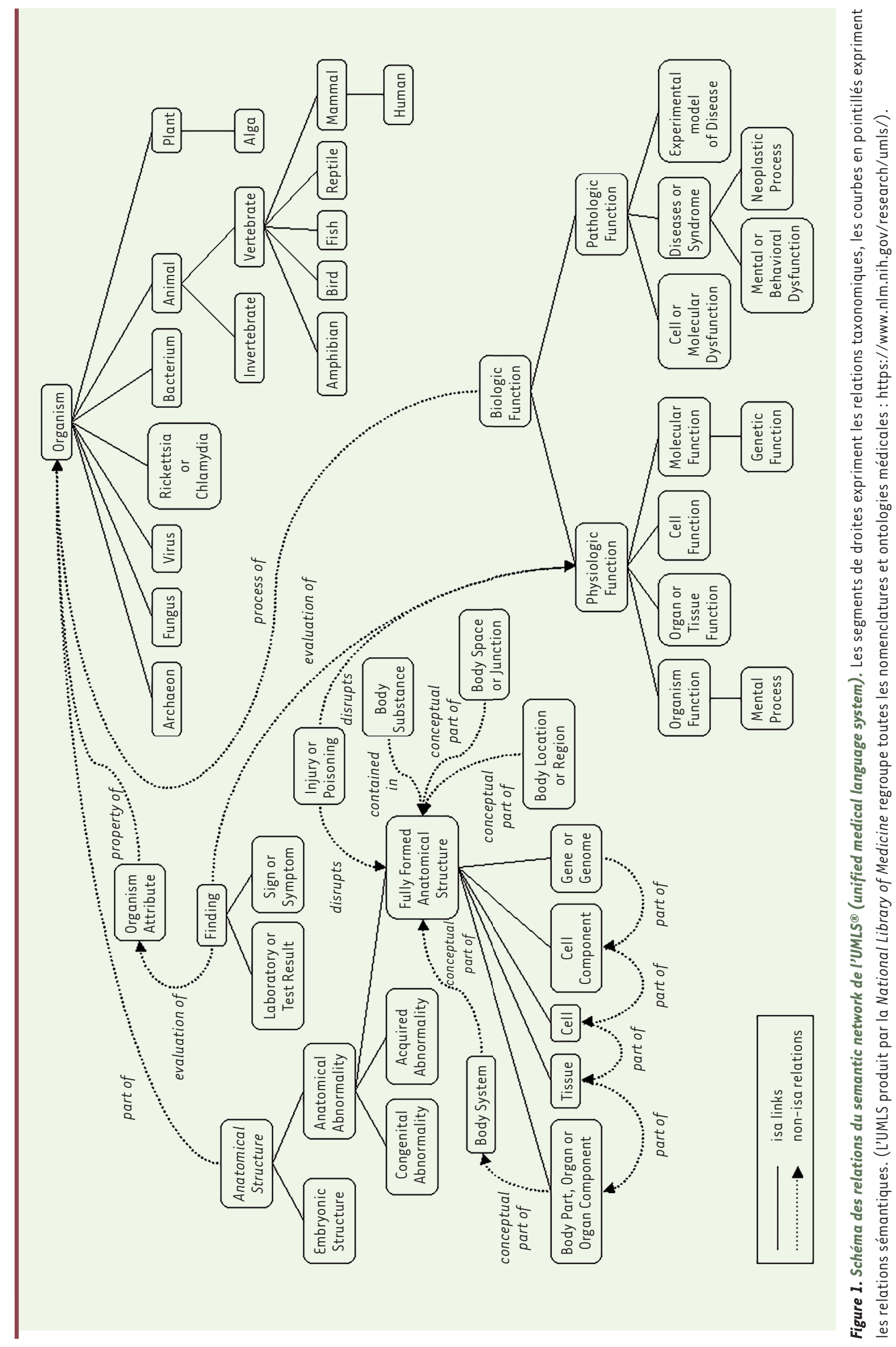




\begin{tabular}{lccc}
\hline & Priméité & Secondéité & Tiercéité \\
\hline $\begin{array}{l}\text { Trichotomie I } \\
\text { Signe par rapport à lui même }\end{array}$ & $\begin{array}{c}\text { Qualisigne } \\
\text { dénué d'identité }\end{array}$ & $\begin{array}{c}\text { Sinsigne (signe singulier) } \\
\text { appliqué une fois }\end{array}$ & $\begin{array}{c}\text { Légisigne } \\
\text { loi générale }\end{array}$ \\
\hline $\begin{array}{l}\text { Trichotomie II } \\
\text { Signe par rapport à l'objet }\end{array}$ & $\begin{array}{l}\text { Icône } \\
\text { ressemble à l'objet }\end{array}$ & Indice & Symbole \\
\hline $\begin{array}{l}\text { Trichotomie III } \\
\text { Signe par rapport à }\end{array}$ & Rhème (terme) & Decisigne (signe qui dit) & loi se substituant à l'objet \\
\hline l'interprétant & interprétable comme un terme \\
\hline
\end{tabular}

Tableau I. Les trois trichotomies du signe.

présentons un extrait des graphes du «semantic network» de l'UMLS (unified medical language system). Ces graphes dans lesquels les relations taxonomiques sont représentées par des segments de droites, regroupent l'ensemble des classifications médicales (Figure 1).

La secondéité implique, elle, une relation entre deux éléments d'un monde dans lequel les objets ne sont plus isolés. L'objet a alors un poids, une couleur. C'est un fait de la vie pratique, elle définit l'existence. Sur le plan de la représentation graphique, c'est un réseau sémantique organisé autour de prédicats à deux places, ou corrélat «... relatif à ... », dans lequel la relation factuelle est variée : «le cœur appartient au thorax», "le sang est de couleur rouge », etc. Dans l'extrait du «semantic network » que l'on présente, il s'agit de relations étiquetées représentées par des lignes pointillées (Figure 1). La tiercéité est la représentation de deux éléments que se fait un troisième qui les observe. Il s'agit donc d'une interprétation. Sa modélisation est un réseau associatif où l'interprète évalue les relations entre les éléments: «la présence d'une fièvre renforce la possibilité d'une maladie infectieuse », «ligaturer une veine empêche le sang de couler », etc. La tiercéité est la catégorie de la pensée et des croyances. Comme l'a montré Barton [10], il est possible de représenter les indicateurs de performance diagnostique (spécificité, sensibilité, valeurs prédictives) dans les ontologies et donc d'y intégrer les graphes associatifs. Pour illustrer ces graphes associatifs, nous pouvons présenter les relations qui relient les signes et les maladies, dont les étiquettes peuvent, sur une échelle de Likert ${ }^{6}$, venir infirmer ou confirmer le diagnostic (Figure $2 A-B$ ) [11].

Ces trois types de réseau (taxonomiques, sémantiques et associatifs) peuvent être représentés simultanément $[12,13]$. Ce sont ces représentations des connaissances médicales que l'on appelle les ontologies médicales et qui permettent de produire des inférences déductives grâce aux algorithmes de logique formelle.

\section{La sémiotique, ou comment nous connaissons le monde}

À partir des catégories fondamentales qu'il a définies, Peirce a fondé la sémiotique, c'est-à-dire la science des signes. En postulant que

${ }^{6}$ Une échelle de Likert est une échelle d'attitude comprenant 4 à 7 degrés par laquelle on demande à l'individu d'exprimer son degré d'accord ou de désaccord relatif à une affirmation.
I'homme n'a pas accès directement aux choses du monde mais seulement à leur représentation, tout, pour lui, est alors signe. Pour Pierce, le signe est une triade de signification: "Un signe, ou représentamen, est quelque chose qui tient lieu pour quelqu'un de quelque chose [...]. Il s'adresse à quelqu'un, c'est-à-dire crée dans l'esprit de cette personne un signe équivalent, ou peut-être un signe plus développé. Ce signe qu'il crée, je l'appelle interprétant du premier signe. Le signe tient lieu de quelque chose, son objet [...]» [14]. Transposé dans le domaine médical, ce sont les signes qu'une maladie nous donne à voir (c'est le représentamen). Ils produisent dans notre esprit le concept de la maladie, regroupant les diverses connaissances que nous avons d'elle (interprétant) à propos d'une pathologie réelle, dont nous ne connaîtrons jamais tous les signes (objet). Ce que I'on retient de la sémiotique de Peirce est généralement la «trichotomie» du signe par rapport à l'objet: icône, indice, symbole. Sa proposition de classification des signes est cependant beaucoup plus riche. Partant de sa définition (représentamen, interprétant, objet) et des trois catégories qu'il a établies (priméité, secondéité, tiercéité), il a en effet proposé une classification du signe en prenant en compte son niveau catégoriel et son rapport à lui-même, à l'objet et à l'interprétant. On obtient ainsi pour un signe, neuf classes : le qualisigne (la pure possibilité du signe : par exemple, jaune), le sinsigne (ce signe-là : Martin a la jaunisse) et le légisigne (la loi qui régit la grammaire du signe : la jaunisse analysée par un médecin); sur le plan de la signification, on aura l'icône (un signe par ressemblance avec l'objet: une photo de conjonctive de patient ictérique, un écran d'ictéromètre), l'indice (un signe relié comme un symptôme à son objet: un taux de bilirubine élevée, une échographie avec des voies biliaires dilatées), et le symbole (un signe doté d'une signification abstraite : les syndromes ictériques connus par les médecins); sur le plan pratique, on aura le rhème (un nom, un verbe, un adjectif : ictère), 
Figure 2. A. Réseau associatif entre signes et maladies. Les relations sont des relations probabilistes: valeurs prédictives positives et négatives. B. Questionnaire de recueil de connaissances sémiologiques du test de concordances des scripts. Dans ce test, on fournit à l'étudiant un contexte clinique; on lui propose alors plusieurs diagnostics et pour chacun des diagnostics, des signes. Charge à lui, ainsi qu'à un panel de correcteurs, de préciser l'impact de ces signes sur l'éventualité du diagnostic dans le contexte clinique. L'étudiant sera évalué par rapport aux réponses du panel de correcteurs et non pas en tout ou rien comme dans un QCM (questionnaire à choix multiples). Plusieurs réponses sont possibles même si elles n'ont pas la même valeur.

B Questionnaire de recueil de connaissances sémiologiques

\begin{tabular}{lll}
$\begin{array}{l}\text { Si vous pensez à } \\
\text { (option diagnostique) }\end{array}$ & $\begin{array}{l}\text { et qu'alors vous trouvez (nouvelle } \\
\text { information obtenue par un examen } \\
\text { clinique ou par un test para clinique) }\end{array}$ & $\begin{array}{l}\text { l'effet sur l'hypothèse diagnostique est } \\
\text { le suivant }\end{array}$ \\
\hline $\begin{array}{ll}\text { Diagnostic } \\
\text { Type d'examen : signe }\end{array}$ & $-2-1+1+2$ \\
\hline l'hypothèse ; + $1:$ l'hypothèse devient plus probable $;+2:$ il ne peut s'agir pratiquement que de cette hypothèse
\end{tabular}

le dicisigne (une proposition verbale ou visuelle: les ictères sont dus à un excès de bilirubine dans le sang) et l'argument (une règle d'inférence : si ictère et dilatation des voies biliaires alors ictère par obstacle biliaire). Pour essayer de donner une traduction des néologismes employés par Peirce, on pourrait proposer que le qualisigne soit un signe élémentaire, le sinsigne un fait, le légisigne une pensée, le rheme un concept, le décisigne une connaissance, et l'argument une inférence.

Il est important d'admettre que ces neuf classes de signes ne sont pas des formes possibles que le signe pourrait prendre dans le phénomène sémiotique, mais des niveaux d'interprétation auxquels un interprète peut être confronté (Tableau I). Si l'on reprend cette classification, par exemple pour une symptomatologie clinique de sciatique, on peut proposer le Tableau /l comme illustration. Sans entrer dans l'algèbre des signes proposée par R. Marty [15], on peut voir dans ce tableau, non seulement une catégorisation du signe mais aussi un processus. Ce processus est orienté de la première case en haut à gauche du tableau (qualisigne) à la dernière case en bas à droite, qui représente la signification la plus aboutie (argument). L'argument peut ensuite, luimême, devenir le qualisigne d'un nouveau tableau dans un processus de communication entre, par exemple, un médecin généraliste et un spécialiste radiologue (Tableau III).

Ce processus peut également être observé lorsque les connaissances médicales progressent. Dans ce cas, Peirce l'appelle le processus de sémiose. La sémiose est la capacité des signes à en produire de nouveaux. Le processus sémiotique est donc, théoriquement, illimité. Dans la pratique, il reste cependant limité... par l'habitude. L'habitude (ou habitus) fige provisoirement le renvoi infini d'un signe à d'autres signes, permettant à des interlocuteurs de s'accorder rapidement sur la réalité dans un contexte donné de communication. Sur le plan culturel, on peut dire que l'habitus est un paradigme au sens de Kuhn
[16]. Sur le plan individuel, c'est le niveau d'expertise de l'interprète $[17,18]$. Sur le plan des signes médicaux, c'est ce qui fera qu'un ensemble de signes (syndrome) deviendra à un moment donné, et dans un contexte défini, un diagnostic de maladie.

\section{L'abduction : notre manière courante de raisonner}

La déduction et l'induction ont été longuement étudiées par les philosophes et les logiciens classiques, bien qu'aucun logicien avant Peirce n'ait reconnu l'importance et la spécificité de cette troisième forme de raisonnement que Peirce a appelé l'abduction. II s'agit pourtant d'une forme de raisonnement mise en œuvre tant dans notre pratique humaine la plus quotidienne, que dans notre pratique médicale courante, que dans la découverte scientifique.

Les caractéristiques de ces inférences sont parfaitement décrites par Peirce à partir de la figure du syllogisme. Prenant le syllogisme déductif comme fondement d'analyse, Peirce constate qu'il est possible d'inverser l'ordre des termes et d'obtenir les différents types d'inférences:

"Par exemple, si en entrant dans une pièce on trouve un sac de haricots blancs et une poignée de haricots posée sur la table, la déduction prend la forme suivante:

- Tous les haricots de ce sac sont blancs (règle).

- Ces haricots proviennent du sac (cas particulier).

- Donc ces haricots sont blancs (conclusion : résultat de la déduction). 


\begin{tabular}{lccc}
\hline & Priméité & Secondéité & Tiercéité \\
\hline $\begin{array}{l}\text { Trichotomie I } \\
\text { Signe par rapport à lui même }\end{array}$ & La douleur & La douleur d'un patient & $\begin{array}{c}\text { La douleur d'un patient } \\
\text { analysée par un médecin }\end{array}$ \\
$\begin{array}{l}\text { Trichotomie II } \\
\text { Signe par rapport à l'objet }\end{array}$ & $\begin{array}{c}\text { Le schéma topographique de la } \\
\text { douleur projetée sur le corps. } \\
\text { L'échelle de la douleur }\end{array}$ & $\begin{array}{c}\text { Les caractéristiques cliniques de } \\
\text { la douleur d'un patient }\end{array}$ & $\begin{array}{c}\text { Les syndromes douloureux } \\
\text { connus par les médecins }\end{array}$ \\
\hline $\begin{array}{l}\text { Trichotomie III } \\
\text { Signe par rapport à } \\
\text { l'interprétant }\end{array}$ & Le terme « sciatique $\$$ & $\begin{array}{c}\text { La sciatique d'un patient avec } \\
\text { Lasègue et impulsive à la toux }\end{array}$ & $\begin{array}{c}\text { Si sciatique impulsive à la } \\
\text { toux et si Lasègue, alors } \\
\text { sciatique discale }\end{array}$ \\
\hline
\end{tabular}

Tableau II. Les trois trichotomies de la sciatique commune.

Une première inversion de ce syllogisme donne pour l'inférence inductive:

- Ces haricots sont blancs (ancien résultat).

- Ces haricots proviennent de ce sac (ancien cas particulier).

- Donc, tous les haricots de ce sac sont blancs (conclusion probable qui tend à rétablir la règle).

Mais, en posant à nouveau la règle comme prémisse majeure, il est une autre inversion possible, celle de la prémisse mineure et de la conclusion de la déduction. On obtient alors l'inférence hypothétique :

- Tous les haricots de ce sac sont blancs (ancienne règle).

- Ces haricots sont blancs (ancien résultat).

- Donc, ces haricots proviennent de ce sac (ancien cas particulier et conclusion hypothétique) » [14] ([2], p. 623).

L'abduction peut aussi s'écrire :

- « Le fait surprenant $C$ est observé.

- Or si A était vrai, C irait de soi.

- Donc il y a une raison de soupçonner que A est vrai » [14] ([5], p. 189).

L'induction peut accroître le degré de confiance que l'on donne à la conclusion (ou la disqualifier). Sachant que les haricots blancs sur la table proviennent du sac, il est probable que tous ceux du sac sont blancs. Pour s'en assurer, il suffit d'en tirer au hasard quelques poignées supplémentaires, augmentant ainsi la probabilité accordée à la vérité de la conclusion. De son côté, l'abduction ne peut faire davantage que de suggérer. Elle crée une hypothèse. Si on médicalise l'exemple, on arrive aux formulations:

- Les rougeoles ont un énanthème (règle).

- Ce patient a une rougeole (cas particulier).

- Ce patient à un énanthème (résultat : déduction).

ou

- Ce patient a une rougeole (ancien cas particulier).

- Ce patient à un énanthème (ancien résultat).

- Les rougeoles ont un énanthème (induction).

ou

- Ce patient à un énanthème (ancien résultat).

- Les rougeoles ont un énanthème (ancienne règle).

- Ce patient a possiblement une rougeole (abduction).
En médecine, à la suite du raisonnement abductif qui a produit l'hypothèse, le raisonnement inductif est ensuite utilisé en recherche clinique, avec les essais cliniques, en utilisant des statistiques sur des échantillons. Ils permettent de définir de nouvelles règles de prise en charge en donnant par exemple la sensibilité et la spécificité ou les valeurs prédictives des signes évalués pour une maladie donnée. La pratique clinique habituelle au lit du malade fait d'abord appel au raisonnement abductif: il s'agit de produire des hypothèses à partir de données incomplètes puis de les évaluer au regard des connaissances acquises sur la valeur des signes mesurés.

Les systèmes d'évaluation des connaissances des étudiants en médecine ont retenu le test de concordance des scripts pour évaluer leur savoir (Figure 2) [19]. Ce test ne permet pas en revanche d'évaluer leur capacité de produire des hypothèses puisque celles-ci leur sont données.

L'abduction a trouvé récemment une nouvelle application avec les données massives, ou big data, et la fouille de données en médecine. Le big data repositionne la recherche empirique par rapport à la recherche contrôlée, cas-témoins. Il s'agit en effet d'utiliser des algorithmes qui, au travers d'observations multiples colligées dans les dossiers patients informatisés, vont permettre de révéler de nouvelles hypothèses causales [20]. Un bon exemple de cette recherche est celle qui a permis de prouver le rôle des glycosides cardiaques comme inducteur de mort cellulaire chez les patients sous chimiothérapie. La fouille de données réalisées chez les patients sous chimiothérapie, entre 1981 et 2009, a en effet permis de révéler une survie supérieure des patients traités par glycosides cardiaques [21]. Cette recherche empirique aurait été impossible pour un seul individu. Elle est au contraire aisée en fouille de données, qui retrouve sur une période de 30 ans les 145 patients ayant reçu une chimiothérapie 


\begin{tabular}{|c|c|c|c|}
\hline & Priméité & Secondéité & Tiercéité \\
\hline $\begin{array}{l}\text { Trichotomie I } \\
\text { Signe par rapport à lui même }\end{array}$ & Sciatique discale & Sciatique discale d'un patient & $\begin{array}{c}\text { Sciatique discale analysée par } \\
\text { un radiologue }\end{array}$ \\
\hline $\begin{array}{l}\text { Trichotomie II } \\
\text { Signe par rapport à l'objet }\end{array}$ & $\begin{array}{l}\text { Image de hernie discale au } \\
\text { scanner }\end{array}$ & $\begin{array}{l}\text { indices de compression } \\
\text { radiculaire au scanner }\end{array}$ & $\begin{array}{l}\text { Les syndromes radiologiques } \\
\text { de compressions radiculaires }\end{array}$ \\
\hline $\begin{array}{l}\text { Trichotomie III } \\
\text { Signe par rapport à } \\
\text { l'interprétant }\end{array}$ & La sciatique par hernie discale & $\begin{array}{l}\text { Hernie discale en contact avec } \\
\text { la racine }\end{array}$ & $\begin{array}{l}\text { Si sciatique d'origine discale } \\
\text { probable et hernie discale au } \\
\text { contact de la racine, alors } \\
\text { confirmation }\end{array}$ \\
\hline
\end{tabular}

Tableau III. Les trois trichotomies de la sciatique d'origine discale.

et des digitaliques. Bien évidemment, reste ensuite à soumettre cette conjecture aux contrôles déductifs et inductifs pour lui permettre d'accéder véritablement au rang de connaissance scientifique et, par là même, à celui de croyance scientifique stable et propice à l'action.

\section{Le pragmatisme ou comment nous pouvons et devons agir sur le monde}

Au sens large, le pragmatisme consisterait à rechercher le sens d'une hypothèse, ou de n'importe quelle idée, dans ses conséquences pratiques que l'on observe. La logique du pragmatisme n'est autre que celle de l'abduction. La philosophie du pragmatisme est la sémiotique : en effet, comme le fait remarquer $\varepsilon$ co [22], Peirce affirme que l'interprétant peut ne pas être un signe mais une action ou un comportement ; par cette position, il fait entrer toute la pragmatique dans le domaine de la sémiotique.

Pour Peirce, la principale tendance de l'homme est de générer de la croyance et, en cela, il s'oppose à Descartes pour qui la principale tendance de l'homme est le doute. Ne pouvant accéder au vrai, l'homme est donc condamné à sans cesse vérifier ses hypothèses. C'est son texte «comment rendre nos idées claires?» [23] qui est considéré comme le point de départ de ce mouvement de philosophie des sciences que l'on retrouvera chez Kuhn et Popper. En effet, falsifiabilité et paradigme sont les mots clés du courant peircien : on ne parle de science que si on peut mettre en cause les conclusions d'une théorie. La physique newtonienne peut ainsi être remplacée par la physique einsteinienne, dès lors qu'elle explique des phénomènes non compris par la première. Inversement, axiome et théorème sont les mots clés du courant de pensée de Descartes : si on part d'un axiome vrai et que la démonstration est prudente, on doit arriver au vrai. Toute la géométrie euclidienne part ainsi du postulat d'une droite, d'un point et d'une parallèle. Cependant, on sait maintenant que l'on peut avoir besoin de géométries non euclidiennes pour expliquer certains phénomènes. En conclusion, la différence fondamentale de la méthode cartésienne et peircienne se résume à ceci : le cartésien cherche à fonder les connaissances en partant de zéro, alors que le projet peircien cherche plutôt à corriger les opinions qui préexistent. C'est évidemment dans cette dernière vision épistémologique que la médecine a un statut de science : la médecine ne repose plus sur la théorie hippocratique des humeurs, mais se construit sur la base de la confrontation anatomo-clinique, de la médecine expérimentale et de l'essai contrôlé. La médecine fondée sur les faits (evidence-based medicine) repose sur ce fondement pragmatique : une théorie médicale est vraie si son application permet de guérir les patients.

C'est par le pragmatisme que Peirce se considérait comme un réaliste. Ce point fut d'ailleurs l'objet de son malentendu sur le pragmatisme avec son ami philosophe William James (1842-1910). Le lien entre sémiotique, pragmatisme et réalisme est cependant incontestable dans la philosophie de Peirce, comme en témoigne les quelques aphorismes repris par Tiercelin [24]: «le pragmatisme n'est en soit aucune doctrine de métaphysique, aucune tentative pour déterminer une quelconque vérité des choses. C'est simplement une méthode pour établir la signification des mots difficiles et des concepts abstraits » [14] ([5], p. 464) ; «l'opinion sur laquelle sont destinés à s'accorder finalement tous ceux qui cherchent, est ce que nous entendons par vérité, et l'objet représenté dans cette opinion est le réel. C'est ainsi que j'expliquerais la réalité » [14] ([5], p. 407).

\section{Conclusion}

Peirce a développé un système de pensée global qui permet de comprendre une discipline aussi complexe que la médecine, tant sur le plan de la représentation de ses concepts, que dans son raisonnement. Les trois catégories de Peirce supportent les trois représentations que I'on retrouve dans les ontologies médicales: les taxonomies, les réseaux sémantiques, les réseaux associatifs. Il est ainsi possible de représenter l'ensemble des connaissances médicales et de les manipuler au travers 
de la logique formelle, ou de les évaluer, par exemple chez un étudiant, avec le test de concordance des scripts.

La sémiotique de Peirce permet de manipuler ces éléments essentiels en médecine que sont les signes, symptômes, syndromes, diagnostics, maladies, en leur donnant un statut allant de la simple qualité à l'argument le plus construit. La sémiose proposée par Peirce permet l'élaboration de nouveaux signes dans le processus de communication ou d'accroissement des connaissances. Le pragmatisme permet de vérifier les concepts. Lorsque le signe se réalise en action, le concept est dit vrai si l'action aboutit. Le système de Peirce est donc réaliste. Ceci est contenu dans le concept de médecine fondée sur les faits. Enfin, l'abduction est l'outil de la sérendipité, c'est-à-dire l'art de trouver fortuitement ce que l'on ne cherche pas. Elle est ainsi l'inférence essentielle de la fouille de données à partir des données massives, ou big data, en médecine. En identifiant de nouvelles hypothèses, elle pourra augmenter le niveau de connaissances médicales. En ce sens, la médecine est identique à l'ensemble de la science qui évolue par réfutations successives des connaissances antérieures. $\diamond$

\section{SUMMARY}

Contribution of C.S. Peirce thought to the representation knowledge and medical reasoning

Modelling knowledge and medical reasoning can be an epistemological project especially now, as medicine seems to reach a scientific status. Through his work on semiotics, abductive reasoning and pragmatism, CS Peirce (1839-1914) offers a series of original solutions. These solutions can give an account of (be considered as a theorical ground for) most of the medical activity in various fields such as the evaluation of the students, the knowledge bases and expert systems, the formal descriptions languages (ontologies), and the evidence-based medicine. By mean of this article, we aim at introducing the medical doctors to this complex but lighting thought on a profession which now uses all the most modern resources of knowledge engineering. $\diamond$

\section{LIENS D'INTÉRÊT}

Les auteurs déclarent n'avoir aucun lien d'intérêt concernant les données pubiées dans cet article.

\section{RÉFÉRENCES}

1. Peirce CS. Textes anticartésiens. Présentation et traduction de Joseph Chenu. Notice biographique. Paris : Aubier, 2004 : p. 5-9.
2. Peirce CS. Textes anticartésiens. Présentation et traduction de Joseph Chenu. Une philosophie de la discursivité radicale. Paris : Aubier, 2004 : p. 11-7.

3. Burnum JF. Medical diagnosis through semiotics: giving meaning to the sign. Ann Int Med 1993 ; 119 : 939-43.

4. Wackenheim $A$. Le traitement des signes. La sémiotique. In : Wackenheim $A$, Zöllner G (eds). Informatique et imagerie médicale. Paris : Masson ; 1995 : p. 39-81.

5. Nessa J. About signs and symptoms: can semiotics expand the view of clinical medicine? Theor Med $1996 ; 17: 363-77$

6. Charlin B, Tardif J, Boshuizen HPA. Script and medical diagnosis knowledge: theory and applications for clinical reasoning instruction and research. Acad Med $2000 ; 75: 182-90$.

7. Upshur R. Certainty, probability and abduction: why we should look to CS Peirce rather than Gödel for a theory of clinical reasoning. J Eval Clin Pract $1997 ; 3: 201-6$.

8. Aristote. Organon 1-II. Chapitre 4. In : Catégories. Paris : Flammarion, 2007 : 370 p.

9. Kant $\varepsilon$. Des concepts purs de l'entendement ou des catégories. In : Critique de la raison pure. Paris : PUF, 1965 : p. 92-9.

10. Barton A, Ethier JF, Duvauferrier R, Burgun A. An ontological analysis of medical Bayesian indicators of performance.J Biomed Semantics 2017 ; 1 : $1-13$.

11. Grumbach A. Cognition artificielle. Paris : Addison-Wesley, 1994 : p. 108-9.

12. Collins AM, Quillian MR. Retrieval time from semantic memory. J Verb Learn Verb Behave $1969 ; 8: 240-47$.

13. Bertaud V, Belhadj I, Dameron 0, et al L'informatisation du signe radiologique.J Radiol $2007 ; 88: 27-37$.

14. The collected papers of CS Peirce. Vol. 1-6. In : Harsthorne C, Weiss P (1931 1935) (eds). Vol. 7-8 In : A. Burks (1958) (ed). Cambridge, MA : Harvard University Press.

15. L'algèbre des signes. Foundations of semiotics. Amsterdam-Philadelphie : John Benjamins, $1990: 406$ p.

16. Kuhn T. La structure des révolutions scientifiques. Paris : Flammarion, 1983 $284 \mathrm{p}$.

17. Eco U. Lector in fabula. Paris : Grasset, 1985.

18. Morand B. Logique de la conception. Figures de sémiotique générale d'après Charles S. Peirce. Paris : L'Harmattan, 2004 : p. 196.

19. Charlin B, Tardif J, Boshuizen HPA. Script and medical diagnosis knowledge: theory and applications for clinical reasoning instruction and research. Acad Med $2000 ; 75: 182-90$.

20. Iranzo V. Abduction and inference to the best explanation. Theoria 2007 ; $60: 339-46$.

21. Menger L, Vacchelli $\varepsilon$, Adjemian S, et al. Cardiac glycosides exert anticance effects by inducing immunogenic cell death. Sci Transl Med $2012 ; 4$ : 143 99.

22. Eco U. Le Signe. Bruxelles : Labor, 1988 : p. 254

23. Peirce CS. Textes anticartésiens. Présentation et traduction de Joseph Chenu. Comment rendre nos idées claires. Paris : Aubier, 1984 : p. 287-308.

24. Tiercelin C. CS Peirce et le pragmatisme. Paris : PUF Philosophie, 1993, 124 p.

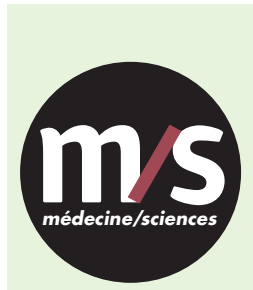

Tarifs d'abonnement $\mathrm{m} / \mathrm{s}-2018$

Abonnez-vous

à médecine/sciences
$>$ Grâce à $\mathrm{m} / \mathrm{s}$, vivez en direct les progrès des sciences biologiques et médicales

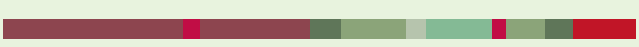

Bulletin d'abonnement page 890 dans ce numéro de $\mathrm{m} / \mathrm{s}$
TIRÉS À PART

R. Duvauferrier
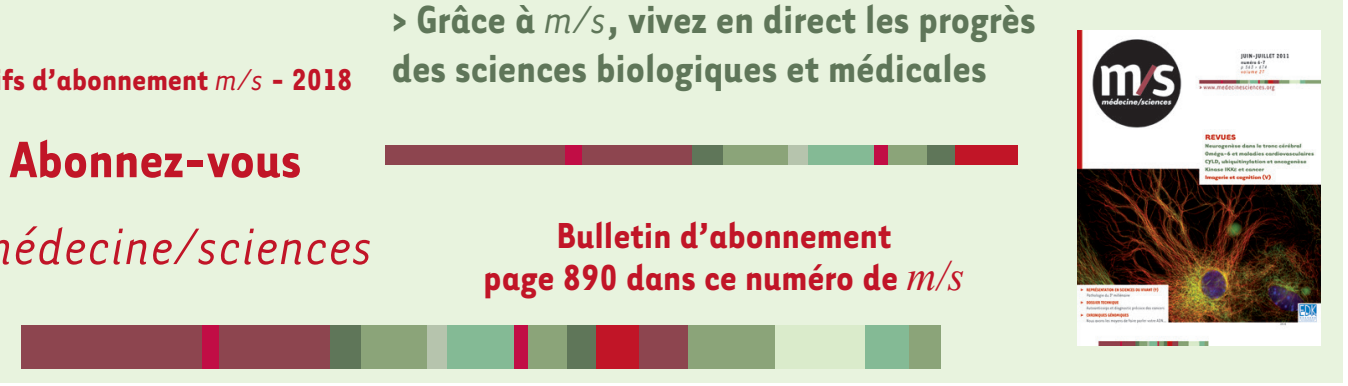